\title{
Image Segmentation of Field Crops Based on the fusion of Infrared and Visible Images
}

\author{
Wang Jian ${ }^{\text {a* }}$ \\ ${ }^{a}$ College of Biology and Science \\ Sichuan Agricultural University \\ Yaan 625014, Sichuan, China \\ Email:wjwj8@163.com
}

\author{
Du ShiPing a \\ ${ }^{a}$ College of Biology and Science \\ Sichuan Agricultural University \\ Yaan 625014, Sichuan, China
}

\begin{abstract}
Efficient and reliable image segmentation \& identification is the basis of scientific crop management and a key technology for precision agriculture. This paper introduces an image segmentation algorithm based on the fusion of infrared and visible images. First of all, the fusion algorithm, which is based on the second-generation Curvelet transformation, was used to fuse the infrared and visible images, and then a cross validation was conducted on the areas of interest on the images to eliminate the interference of background and finally, by taking the estimated contour of the treated target as the initial growth curve, the dynamic edge evolution technology was employed to fix the edge of the target and complete the segmentation. According to the experimental results, this algorithm can effectively fuse the important information in the visible and infrared images without damaging the shape characteristics of crops, thus achieving better segmentation results.
\end{abstract}

Keywords: crops, image segmentation, infrared image, visible image, the second-generation Curvelet transformation, image fusion.

\section{INTRODUCTION}

Precision agriculture refers to the agricultural production \& management technology conducting quantitative investment in the crop-growing areas. The key point of precise control rests with the accurate acquisition and identification of information about the field crops, that is, identifying the crops from the complex background. With the development of photoelectric sensor and image processing technology as well as the deepening of people's understanding of the characteristics of agricultural crops, some studies on the processing and application of infrared images have been carried out. Infrared image processing technology with the existing visible image processing technologies can be used in crop management, identification of field crops and weed, harvest machinery and its vision navigation and quality grading to make up the disadvantages of the current visible image processing technology[1][2], thus overcoming the current problems like low detection rate and precision.

Crop segmentation is the precondition for the following acquisition and identification of crop features, and thus the quality of segmentation is quite important. The segmentation of targets in complex conditions will be interfered by many factors, such as the background, difference in color distribution on the targets, etc, while it's rather difficult to acquire satisfactory results if only using the traditional methods for segmentation. Reflecting the thermal radiation information of the targets, infrared images are almost not affected by the lighting conditions and perform well when the lighting is poor, and they are good at reflecting the targets with different degrees of radiation in the same background. On the other hand, with rich gray-scale distribution, visible images can reflect the light reflection characteristics of crops and contain the abundant details like the edge and texture of crops, but they are easily affected by the lighting conditions[3]. The fusion of images acquired by infrared and visible sensors is an effective way to combine the target characteristics in infrared images with the crop details in visible images.

In our experiment, a fast discrete Curvelet transformation was conducted on the visible and infrared images of the original crops to work out the sub-band coefficients at different dimensions and directions. For low-frequency sub-band coefficients, the fusion weight was determined in accordance with the crop features in the infrared images and the detail information in the visible images. For high-frequency sub-band coefficients, the fusion rules based on local energy match would be adopted. Finally, the fusion results were acquired after undergoing the inverse Curvelet transformation. Then, a cross validation was conducted on the areas of interest on the images to eliminate the interference of background and finally, by taking the estimated contour of the treated target as the initial growth curve, the dynamic edge evolution technology was employed to fix the edge of the target and complete the segmentation. 


\section{THE SCHEME FOR FUSION OF INFRARED AND VISIBLE IMAGES OF CROPS}

First of all, using Curvelet[4], the infrared and visible images of crops were transformed to work out the Curvelet coefficients of the two original images at different scales and directions. The two Curvelet coefficients are $\left\{C_{j_{0}}^{I R}\left(k_{1}, k_{2}\right), C_{j, l}^{I R}\left(k_{1}, k_{2}\right)_{j \geq j_{0}}\right\} \quad$ and $\left\{C_{j_{0}}^{V}\left(k_{1}, k_{2}\right), C_{j, l}^{V}\left(k_{1}, k_{2}\right)_{j \geq j_{0}}\right\}$

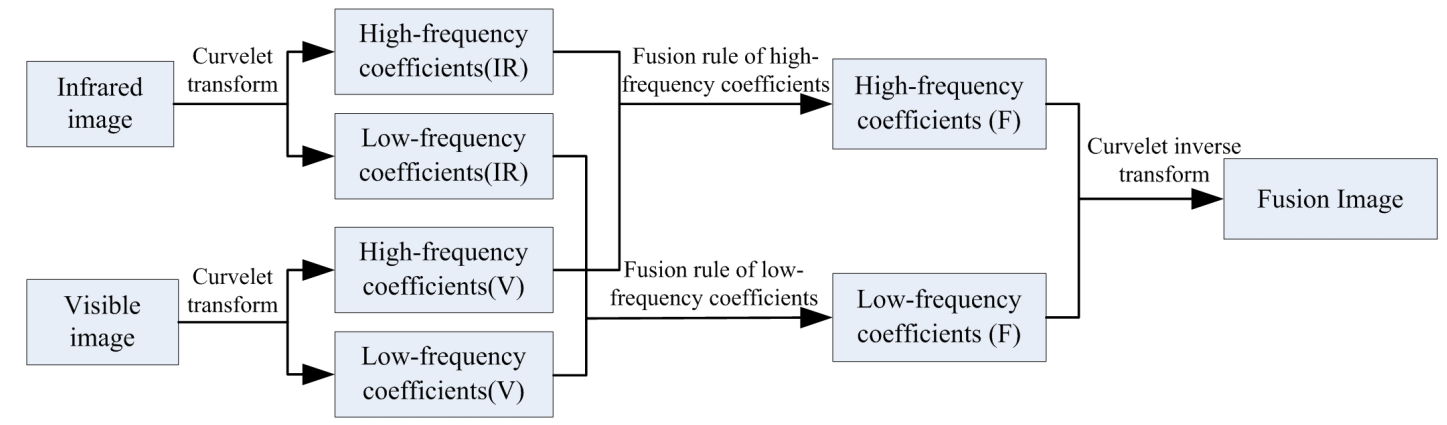

Figure 1. Fusion scheme of infrared and visible images based on Curvelet transform

\section{A. The rules for fusion of low-frequency coefficients}

In the Curvelet transformation of images, the lowfrequency subband, which contains the main energy of the images, contributes quite a lot to the quality of fusion. On the other hand[5], due to the difference in imaging mechanism between the infrared and visible sensors, the gray distribution of the crops in one image is quite different from that in the other image. Thus, the traditional fusion rules with weighted mean are not competent enough in dealing with the low-frequency coefficients. For this reason, we introduced a set of rules to fuse the low-frequency coefficients in accordance with the physical characteristics of the infrared and visible sensors.

The thermal radiation of the targets in the infrared images is obvious, and its grey value is usually significantly higher than that of the background. We conducted a cluster analysis on the low-frequency subband coefficients in the infrared images, and then defined that the point belongs to the normalized confidence of the target.

$$
\omega^{I R}\left(k_{1}, k_{2}\right)=\frac{\max \left\{\left[C_{j_{0}}^{I R}\left(k_{1}, k_{2}\right)-T^{I R}\right], 0\right\}}{\max _{M, N}\left[C_{j_{0}}^{I R}\left(k_{1}, k_{2}\right)\right]-T^{I R}}
$$

In this formula, $\mathrm{M}$ and $\mathrm{N}$ represent the size of the lowfrequency sub-band, while $T^{I R}$ represents the segmentation threshold.

Since there is usually significant difference in area between the target and background in the infrared images, great errors may occur while using the traditional cluster analysis methods like Otsu algorithm. To solve this problem, we used the partial Otsu recursive segmentation algorithm to capture the target area, and the basic principle is: after conducting an Otsu segmentation on the original image, the treated image will contain two parts (the target area and background); if the target area still
After that, we adopted different approaches to fuse the low-frequency and high-frequency coefficients of the two images in accordance with the physical properties of the infrared and visible sensors, and then acquired the Curvelet coefficients of fusion at different directions, namely, $\left\{C_{j_{0}}^{F}\left(k_{1}, k_{2}\right), C_{j, l}^{F}\left(k_{1}, k_{2}\right)_{j \geq j_{0}}\right\}$. Finally, we conducted an inverse Curvelet transformation and got the fused image $I^{F}$. The scheme for fusion of infrared and visible images is shown in Figure.1. contains a part of background, we will continue to conduct Otsu segmentation until acquitting satisfactory images. Upon the completion of an Otsu segmentation[6], the algorithm will abandon a number of background pixel sets with low grey level, which can not only reduce the calculation for the next segmentation but also minimize the impact of the number of pixels on the segmentation results. On the other hand, visible images can reflect the edge and texture of the scene, so we worked out the gradient value $G^{V}$ of each point in the low-frequency part of the visible images and then conducted a normalized processing.

$$
\omega^{V}\left(k_{1}, k_{2}\right)=\frac{G^{V}\left(k_{1}, k_{2}\right)}{\max _{M, N}\left[G^{V}\left(k_{1}, k_{2}\right)\right]}
$$

In summary, the normalized measurement degree $\omega^{I R}$ and $\omega^{V}$ that are based on physical properties reflect the detection features and edge details of targets, respectively. Thus, the rule for fusion of the low-frequency sub-band coefficients can be described by the following formula:

$$
\begin{gathered}
C_{j_{0}}^{F}\left(k_{1}, k_{2}\right)=\alpha C_{j_{0}}^{I R}\left(k_{1}, k_{2}\right)+(1-\alpha) C_{j_{0}}^{V}\left(k_{1}, k_{2}\right) \\
\text {, in which } \alpha=\frac{\omega^{I R}\left(k_{1}, k_{2}\right)}{\left[\omega^{I R}\left(k_{1}, k_{2}\right)+\omega^{V}\left(k_{1}, k_{2}\right)\right]}
\end{gathered}
$$

\section{B. The rules for fusion of high-frequency coefficients}

For the high-frequency sub-band coefficients of the infrared and visible images at different scales and directions, we adopted the fusion rules based on the local area energy match. First of all, the measurement degree of local area energy of every element in the high-frequency 
subband of the infrared and visible images was worked out.

$$
E_{j, l}\left(k_{1}, k_{2}\right)=\sum_{s \in S, t \in T}\left|C_{j, l}\left(k_{1}+s, k_{2}+t\right)\right|^{2}
$$

In this formula, $\mathrm{S}$ and $\mathrm{T}$ represent the sizes of the local areas, and the matching degree corresponding to every element in the high-frequency subband of the infrared and visible images was worked out, respectively.

$$
M_{j, i}^{I R, V}\left(k_{1,} k_{2}\right)=\frac{2 \sum_{s \in S, t \in T}\left|C_{j, l}^{I R}\left(k_{1}+s, k_{2}+t\right) C_{j, l}^{V}\left(k_{1}+s, k_{2}+t\right)\right|}{E_{j, l}^{I R}\left(k_{1}, k_{2}\right)+E_{j, l}^{V}\left(k_{1}, k_{2}\right)}
$$

In this formula, $T^{M}$ represents the default matching threshold, and then the high-frequency sub-band coefficient in the fusion results is:

$$
C_{j, i}^{F}\left(k_{1}, k_{2}\right)=C_{j, l}^{V}\left(k_{1}, k_{2}\right) \quad, \quad E_{j, i}^{V}\left(k_{1}, k_{2}\right) \geq E_{j, i}^{I R}\left(k_{1}, k_{2}\right)
$$

or

$$
C_{j, i}^{F}\left(k_{1} k_{2}\right)=C_{j, l}^{I R}\left(k_{1} k_{2}\right) \quad, \quad E_{j, i}^{V}\left(k_{1}, k_{2}\right)<E_{j, i}^{I R}\left(k_{1}, k_{2}\right)
$$

\section{EXTRACT THE CONTOUR OF THE TARGET CROPS WITH DYNAMIC EDGE EVOLUTION TECHNOLOGY}

The traditional object segmentation algorithm based on threshold or the edge information in images cannot get rid of the terrible interference of background. Especially, for the visible images with complex backgrounds, the mistakes in capturing background areas often occur due to the sharp changes of backgrounds. To eliminate the impact of the complex background, we adopted dynamic edge evolution technology to capture the contour of the objects.

The dynamic edge evolution technology can define an energy function[7] (independent variables include the boundary contour curve) by use of the grey distribution of the targets and background in the images. After that, we acquired the curve evolution equation matching the energy function through the dynamic formula of EulerLagrange equation. To speed up the calculation, we employed the level set method to simulate the evolution of the initial curve towards the direction, along which the energy decreases most sharply, and then worked out the optimal boundary contour curve. The area-based dynamic edge evolution technology for image segmentation can take the initiative to use all the light intensity information inside and outside the contour and avoid the sensitivity to noise while using gradient-based edge detection functions, thus performing well while dealing with the images interfered by noises. The energy equation based on Mumford-Shah function, which was developed by Chan and Vese[8], is good at coping with the segmentation of images with different mean areas of the objects and background, and the energy function is as below:

$$
\begin{aligned}
\operatorname{Min}_{C} E(C(P))= & \alpha \iint_{\Omega_{\text {in }}}\left|I(x, y)-c_{1}\right|^{2} d x d y \\
& +\beta \iint_{\Omega_{\text {out }}}\left|I(x, y)-c_{2}\right|^{2} d x d y+\mu \oint_{C} d s
\end{aligned}
$$

In this function, $c_{1}$ is the inner mean value of targets determined by the contour $\Omega_{\text {in }}$ in the image $I ; c_{2}$ is the mean value of the background area determined by $\Omega_{\text {out }}$; the last term represents the length of the boundary curve; $\alpha, \beta, \mu$ are the weight factors of the corresponding items. In the evolution, the curve $C(t)$ is expressed as the zero level set of the level set function $\phi(x, y, t)$, which is then introduced into Formula (8) through the Heaviside function $H(\phi)$, and then we need to convert the mixed integral of the area and boundary into a single integral of the boundary. The minimum solution is achieved using the Euler-Lagrange equation that is shown as below:

$$
\begin{gathered}
\frac{\partial \phi}{\partial t}=\delta_{s}(\phi)\left[\mu \cdot \operatorname{div}\left(\frac{\nabla \phi}{\|\nabla \phi\|}\right)-\mu-\alpha\left(I(x, y)-c_{1}\right)^{2}\right. \\
\left.+\beta\left(I(x, y)-c_{2}\right)^{2}\right]
\end{gathered}
$$

In this equation, $\delta_{s}(\phi)$ is the regularization function of the Dirac measurement and also the first derivative of $H(\phi)$ function.

\section{EXPERIMENTAL RESULTS AND CONCLUSIONS}

The work process of the target segmentation algorithm to deal with crop images is shown in Figure 2. For the crop images with the background of fields, we need to conduct the extraction of the target areas, the fusion and verification of the target areas, and the dynamic edge evolution, etc. To guarantee that the edge of the target is smooth and complete, we finally found out the edge by taking the initial estimate of the target' contour as the initial growth curve with the help of the dynamic edge evolution technology. 


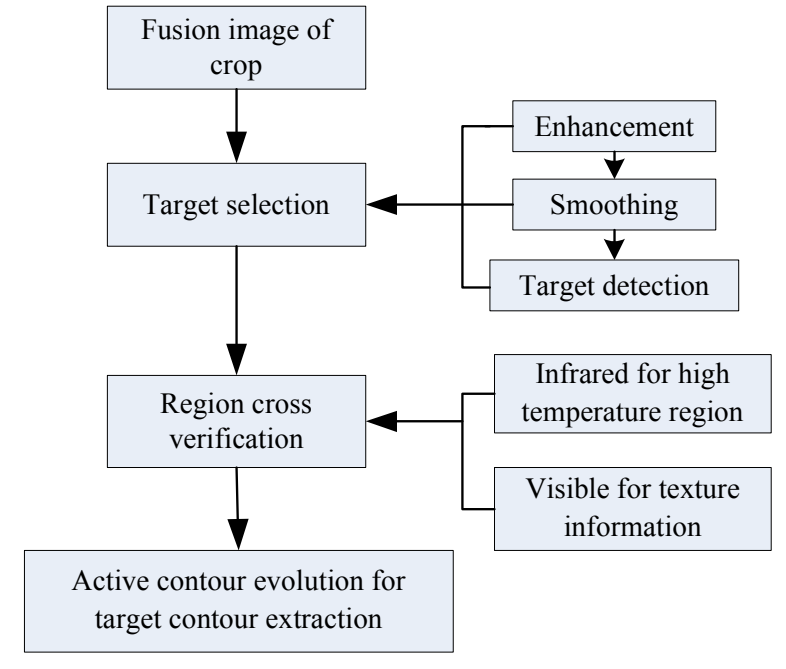

Figure 2. crop images target segmentation process

In this experiment, we took the crop object segmentation algorithm to test the images of the field crops. According to the results, the application of infrared light can effectively overcome the problem that the spectrum effect of green plants in visible light is not obvious. The segmentation on celeries, radishes and wheat (especially on the fruits and flowers) is satisfactory, but that on weeds is still not good enough. Figure 3. shows the infrared and visible images, the fused images and the segmentation results of the wheat grass in the fields.
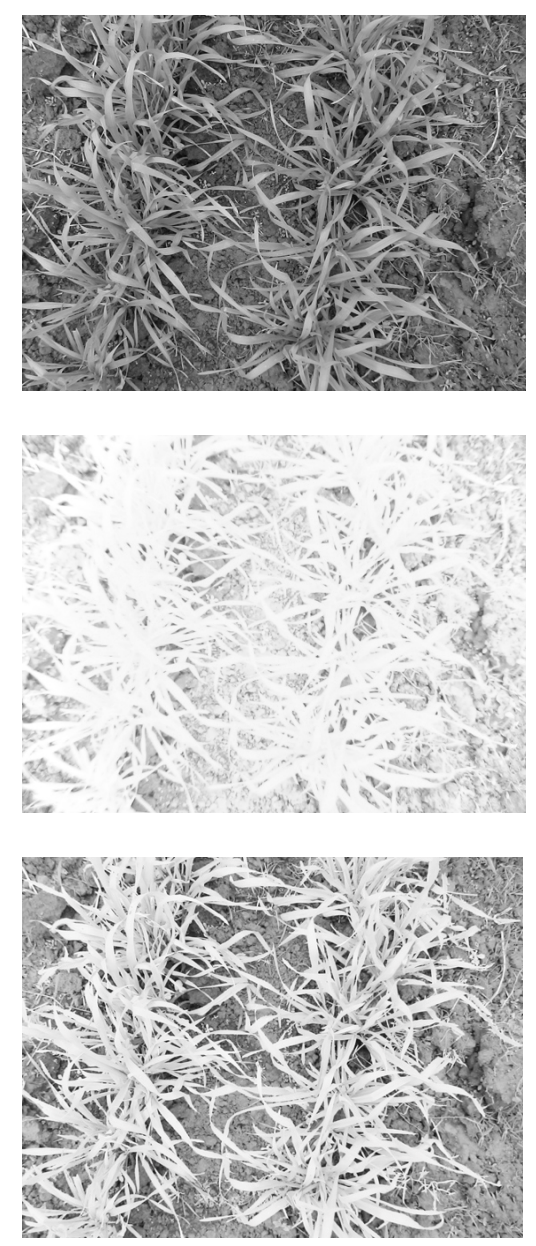

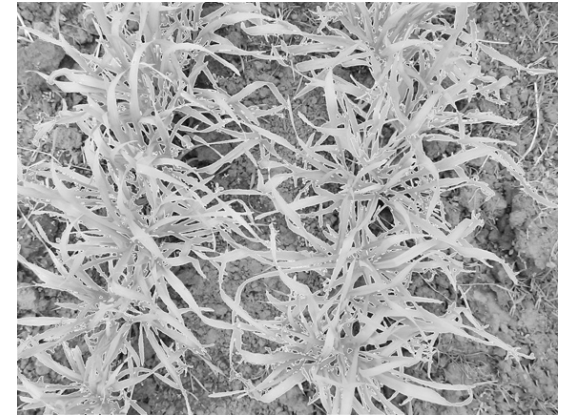

Figure 3. The infrared and visible images, the fused images and the segmentation results of wheat

It is indicated in the experimental results that this algorithm, with the advantages of both infrared and visible images, performs well in maintaining the target features in the infrared images and the detailed information in the visible images, thus achieving an accurate segmentation on crops. Therefore, it can be regarded as an effective segmentation technology that is of great help to the monitoring on the nutritional status and growth of the field crops.

\section{REFERENCES}

[1] Torii T, Kitade S, Teshima T. Crop row tracking by an autonomous vehicle using machine vision (part1)[J]. J of the Japanese Society of Agricultural Machinery, 2000, 62(2): $41-48$.

[2] Takoi K Hamrita Tollner E W , Robert L Schafer. Toward fulfilling the robotic farming vision: advances in sensors and controllers for agricultural applications $[\mathrm{J}]$. IEEE Trans on Industry Applications, 2000, 36(4): 10261032.

[3] Toet A, Ijspeert J K,Waxman A M, Fusion of visible and thermal imagery improve situational awareness[J].Displays,1997,18(2):85-89

[4] Candes E,Demanet L. DonohoD, Fast discrete curvelet transforms [J].Multiscale Modeling \& Simulation,2006,5(3):861-899

[5] Wang Chen-yi, Wang Jian-jun, Information Hiding Method of Second Generation Curvelet Transform, Information and electronic engineering, 2008,6(2), 105-109 (in Chinese)

[6] Otazu X,Gonzalez Audicana M,Fors O, Introduction of sensor spectral response into image fusion methods. Application to wavelet-bases methods [J].IEEE Transactions on Geoscience and Remote Sensing,2005,43(10):2376-2385

[7] Mumford D, Shah J. Optimal approximation by piecewise smooth functions and associated variational problems[J]. Communication on Pure and Applied Mathematics, 1989, 42(5): 577-685.

[8] Chan T F,Vese L. Active contours without edges[J]. IEEE Transactions on Image Processing，2001， 10(2): 266-277 